\title{
Metodologías ágiles
}
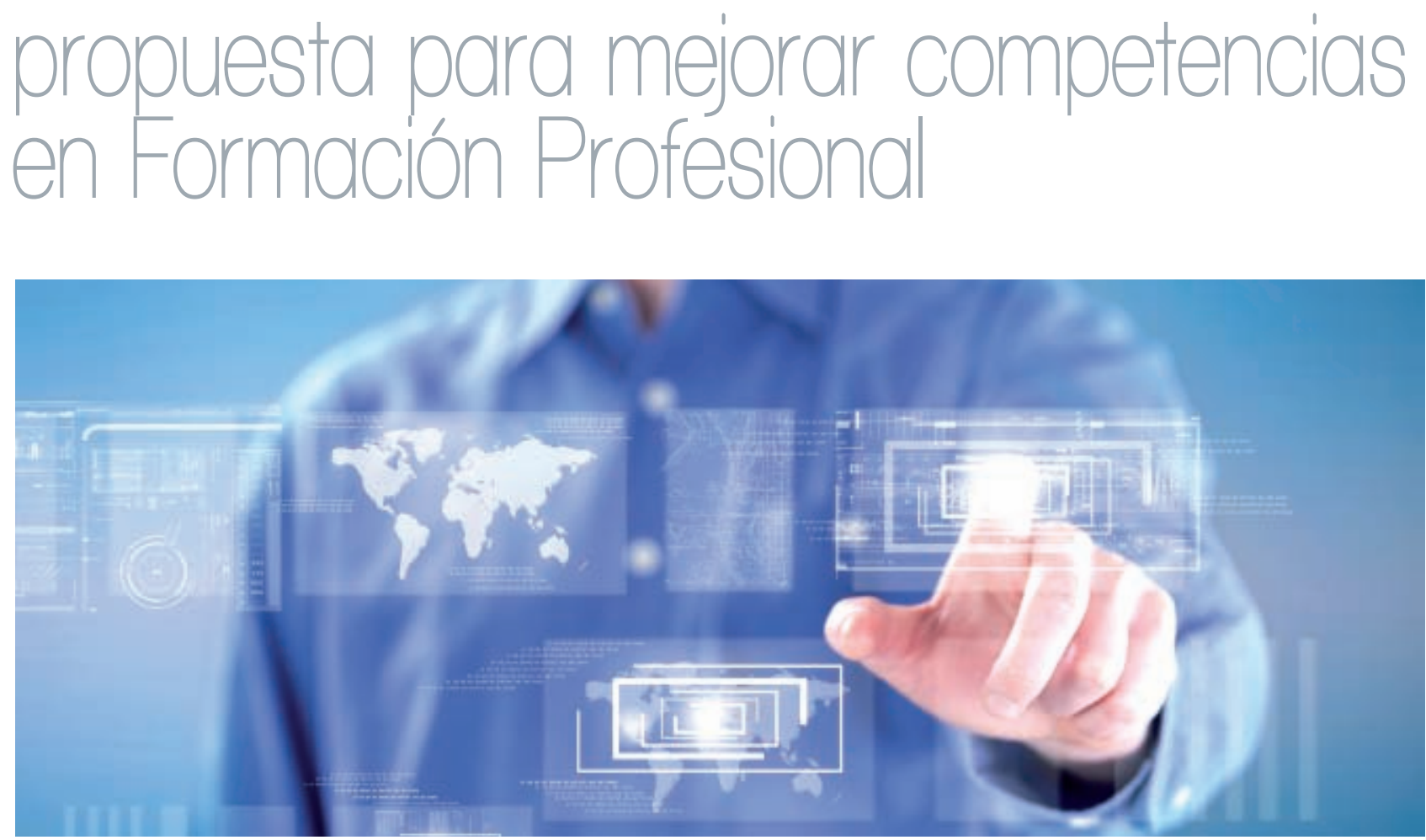

La experiencia llevada a cabo consiste en la integración en el currículum del módulo formativo de Empresa e Iniciativa Emprendedora (EIE) de los nuevos procedimientos metodológicos, globalmente denominados "metodologías ágiles" y

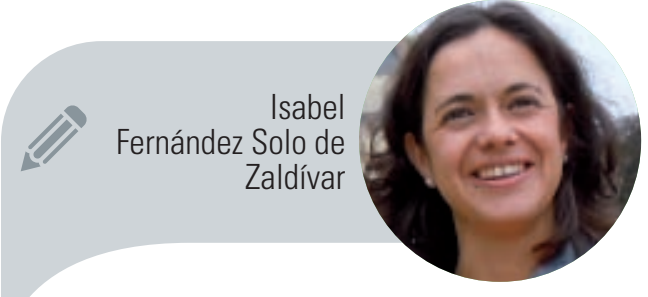

Profesora de la Universidad Internacional de la Rioja isabelfernandezsolo@gmail.com que tanto auge han cobrado últimamente en ámbito empresarial en lo relativo al emprendimiento.

La adaptación de metodologías aplicadas con éxito en otros ámbitos puede ser una vía de investigación y práctica que proporcione resultados óptimos en la búsqueda de empleo y contribuya al desarrollo de algunas de las capacidades consideradas esenciales en la empleabilidad de los jóvenes: la iniciativa, la confianza en sí mismo y la comunicación. 


\section{Introducción}

La Ley Orgánica 2/2006 de 3 de mayo de Educación (LOE) recogía ya entre sus fines conseguir que el alumnado desarroIlara el espíritu emprendedor. La LOE, que entró en vigor en 2009, abrió la puerta a los avances en esta materia, que fue desde ese momento introducida de manera oficial en los reales decretos sobre enseñanzas mínimas de los diferentes ciclos de Formación Profesional.

En cuanto a la Ley Orgánica 8/2013 de 9 de diciembre para la mejora de la calidad educativa (LOMCE), podemos destacar la inclusión de competencias y habilidades alejadas de los contenidos teóricos y también sus reiteradas alusiones al fomento de la a emprendedora

Ya desde su preámbulo, se establece que "el aprendizaje en la escuela debe ir dirigido a formar personas autónomas, críticas, con pensamiento propio". Y más adelante se recoge como lógica y fundamento de las reformas propuestas "la evolución hacia un sistema capaz de encauzar a los estudiantes hacia las trayectorias más adecuadas a sus capacidades, de forma que puedan hacer realidad sus aspiraciones y se conviertan en rutas que faciliten la empleabilidad y estimulen el espíritu emprendedor a través de la posibilidad, para el alumnado y sus padres, madres o tutores legales, de elegir las mejores opciones de desarrollo personal y profesional". Deja claro también que "las habilidades cognitivas, siendo imprescindibles, no son suficientes; es necesario adquirir desde edades tempranas competencias transversales, como el pensamiento crítico, la gestión de la diversidad, la creatividad o la capacidad de comunicar, y actitudes clave como la confianza individual, el entusiasmo, la constancia y la aceptación del cambio".

Establecidos estos principios, se destacan tres ámbitos sobre los que la LOMCE hace especial incidencia con vistas a la transformación del sistema educativo: las tecnologías de la información y la comunicación, el fomento del plurilingüismo, y la modernización de la Formación Profesional.

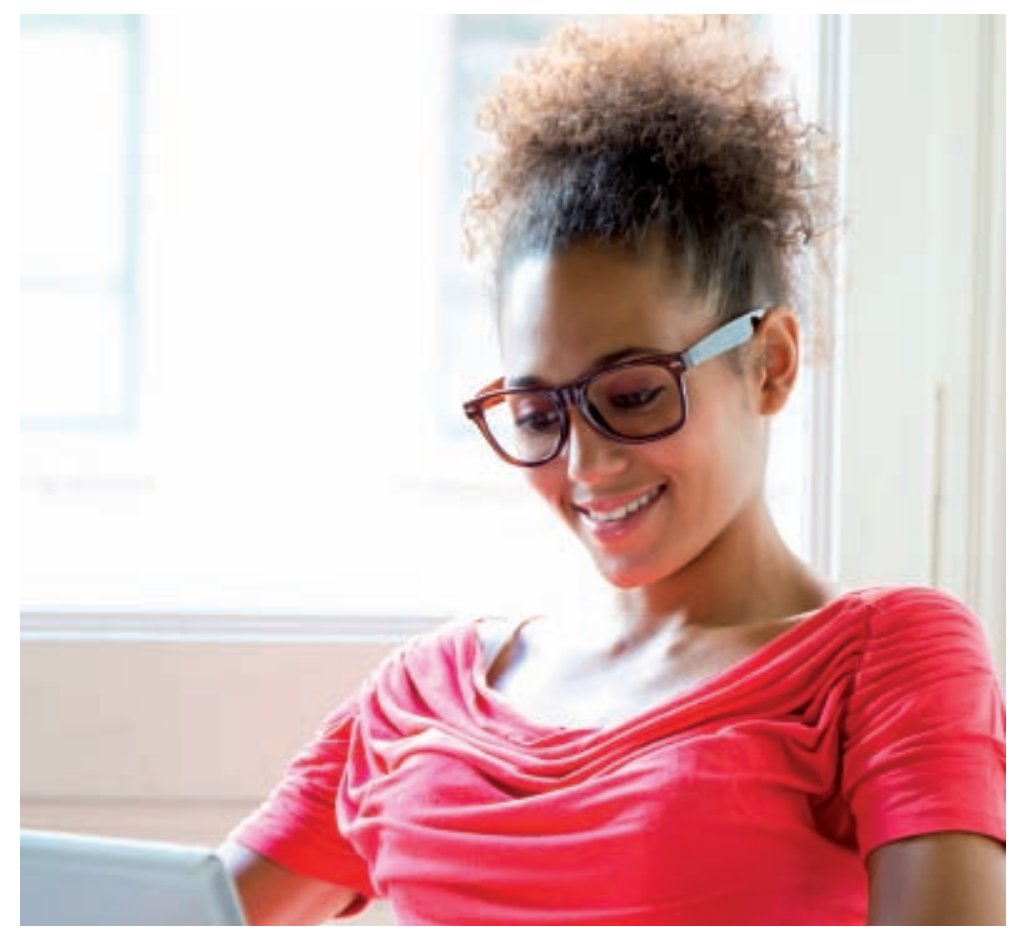

\section{Objetivo y reto}

Para dar una adecuada respuesta a estos retos es importante que el sistema educativo fomente actitudes y comportamientos emprendedores, no solamente para crear nuevas empresas sino con el fin más ambicioso de generar en el individuo unas competencias para ser más creativos, tener más confianza en sí mismos, convertir ideas en actos.

Hasta hace poco tiempo, la tendencia en la formación superior consistía únicamente en formar técnicos y profesionales para los sectores primario, manufacturero o de servicios. Pero actualmente, los nuevos técnicos o profesionales deben contar, además de con los propios conocimientos, habilidades y competencias técnicas propias de su profesión, con una dosis mínima de creatividad intelectual, capacidad analítica y espíritu empresarial, que les permita generar adecuadamente su propio trabajo (Díaz Pérez, 2007). El desarrollo de estas habilidades puede ser una función importante de los nuevos Centros Integrados de Formación Profesional.

\section{Contextualización}

Los diseños curriculares actuales de los ciclos de Formación Profesional y la inclusión en las diferentes normativas sobre educación de conceptos y materias específicas referentes a la empleabilidad 


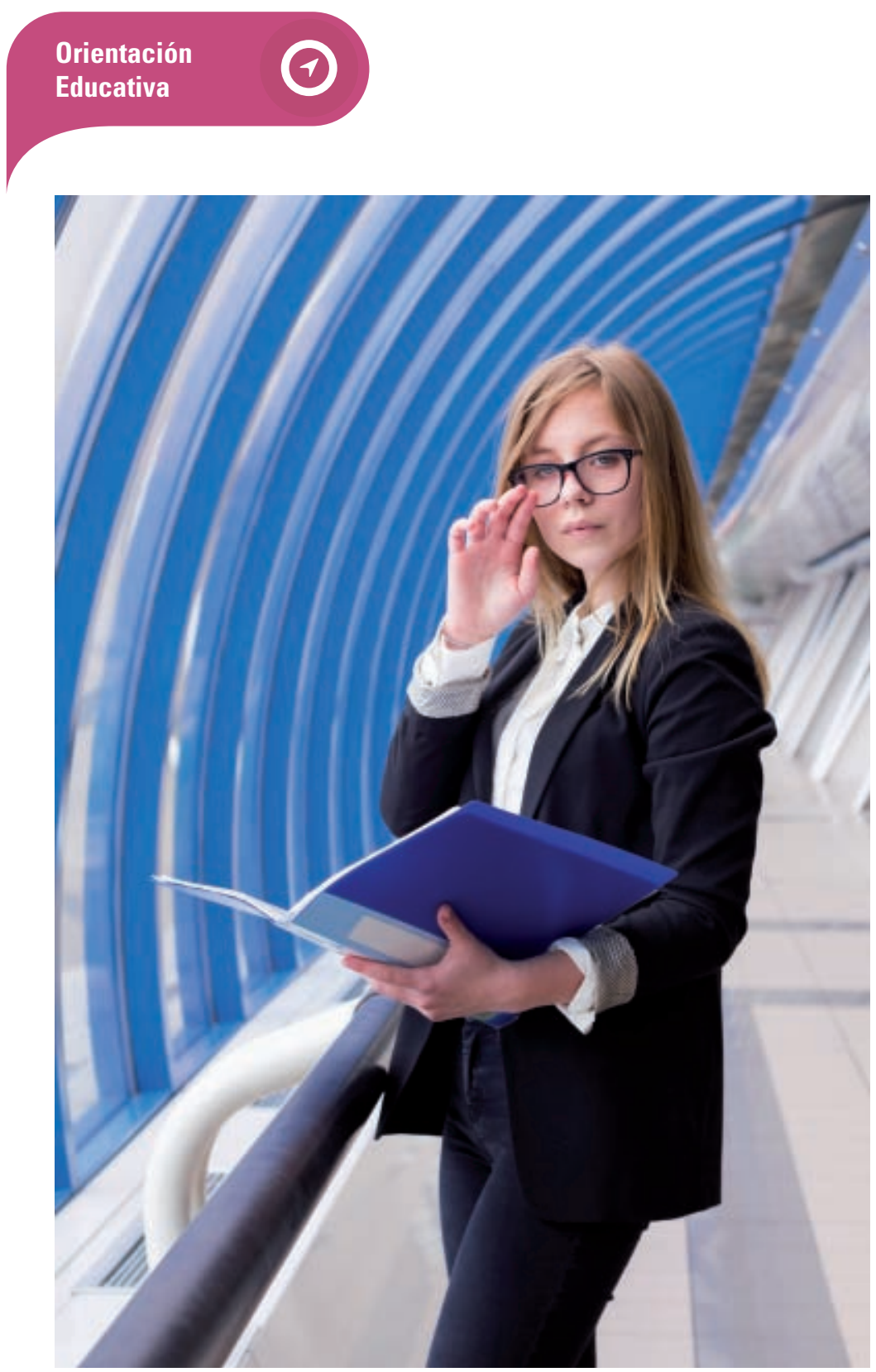

y a la creación de empresa nos presentan un marco idóneo para fomentar el espíritu emprendedor y la empleabilidad en los estudiantes que cursan Formación Profesional, ya que incluyen en todos los ciclos formativos módulos que deben cursarse obligatoriamente: Formación y Orientación Laboral (FOL) y Empresa e Iniciativa Emprendedora (EIE).

A pesar de ello, estudiando la realidad podemos encontrarnos varios problemas:

入 Aunque es cierto que está recogida legalmente en el diseño de todos los ciclos LOE y se incluye Empresa e Iniciativa Emprendedora-EIE como un módulo diferenciado, con frecuencia su desarrollo no está suficientemente enfocado a la adquisición de auténticas competencias emprendedoras, sino más bien se emplea para la enseñanza de conceptos de empresa y normativos de más que dudosa eficacia para un futuro auge del emprendimiento.

7 Existen ciclos de Formación Profesional especialmente dirigidos al ámbito de la administración de empresas donde se incide especialmente en las capacidades empresariales. Pero sin embargo, debemos tener en cuenta que es más probable que las ideas empresariales innovadoras y viables surjan de estudios técnicos, científicos y creativos.

7 Es un hecho que en la actualidad existe un mayor interés social por el fomento de la cultura emprendedora (quizás por la especial coyuntura económica), sin embargo los recursos, tanto humanos como económicos, dedicados desde el ámbito educativo para la adquisición de estas competencias son claramente insuficientes, siendo por tanto imposible responder adecuadamente a esta demanda. La enseñanza orientada a esta acción es laboriosa y costosa, y requiere además una formación específica.

$\boldsymbol{\eta}$ Los departamentos formativos de los centros educativos tienden a trabajar de manera independiente ante una materia que debiera por el contrario trabajarse transversalmente. Partimos de unas estructuras basadas en unos planes de estudios rígidos que constituyen a menudo en sí mismas un obstáculo para la introducción de enfoques interdisciplinares.

त Un aspecto especialmente relevante son las metodologías de enseñanza utilizadas. Existen una amplia gama de técnicas para sustituir a las clases magistrales como herramienta básica de enseñanza. Sin embargo, parece existir un desajuste entre los métodos utilizados en la práctica y los que se consideran más eficaces y apropiados. Los métodos de enseñanza tradicionales, como las lecciones magistrales, evidentemente no son los más adecuados para desarrollar una mentalidad creativa y emprendedora. 


\section{Una propuesta metodológica en la Formación Profesional navarra}

Existen numerosos estudios y teorías que demuestra que debemos modificar nuestras metodologías de enseñanza, hacia otras más acordes con la sociedad cambiante que en la que nos movemos. La pirámide de aprendizaje de Edgar, y el concepto cada vez más en auge del learning by doing, son buenas bases para el desarrollo y aplicación de nuevas metodologías en todas las áreas curriculares.

Debemos responder, por lo tanto, con un enfoque didáctico integral, por lo que el resultado del proceso de aprendizaje debe ser un equilibrio entre "la cabeza, el corazón y la mano" (Johann Heinrich PestalozzI, 1746-1827), es decir, del aprendizaje cognitivo, afectivo y psicomotriz. La enseñanza orientada a la acción se inicia en una situación concreta propia de cada alumno que debe ser ejercitada para luego deducir una regla general.

Para alcanzar esto tenemos a nuestra disposición nuevas propuestas metodológicas especialmente orientadas a estos procesos. En concreto, el Servicio de Formación Profesional del Gobierno de Navarra propone la utilización de las denominadas "metodologías ágiles" como modelo de desarrollo metodológico adecuado a los alumnos que cursan ciclos formativos de Formación Profesional.

\section{Concepto de metodologías ágiles}

El término "métodos ágiles" se utiliza para definir a los métodos de desarrollo empresarial que nace como alternativa a otras metodologías excesivamente rígidas.

Surgen de la necesidad de un nuevo método para las empresas que trabajan en escenarios que necesitan estrategias basadas en la agilidad y la flexibilidad y que precisan adaptarse a un entorno en muchas ocasiones inestable y con un rápido desarrollo.

Este tipo de empresas necesitan construir su producto al mismo tiempo que cambian y aparecen nuevos requisitos. Es evidente que no podemos modificar las circunstancias de los mercados, así se
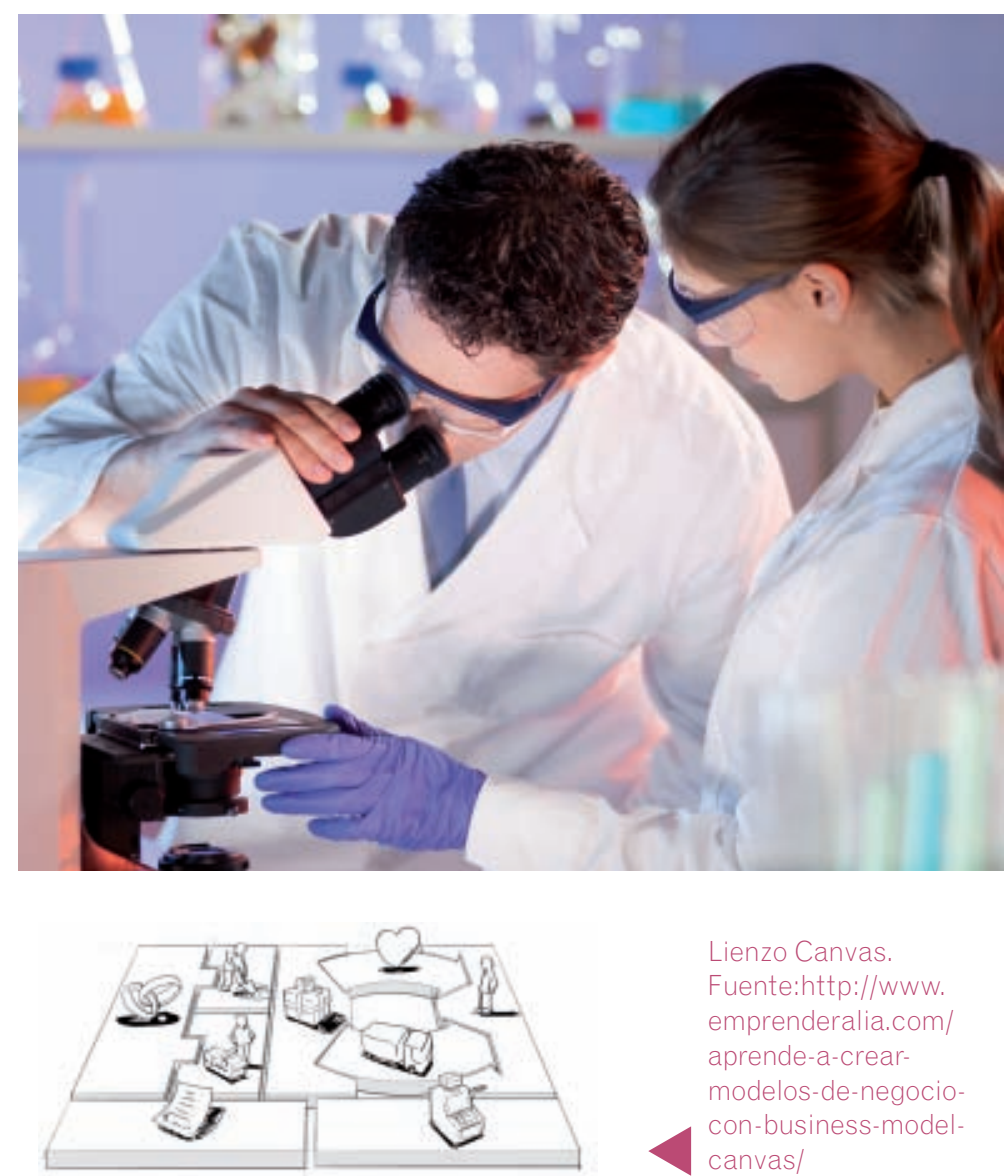

Lienzo Canvas.

Fuente:http://www. emprenderalia.com/ aprende-a-crearmodelos-de-negocio con-business-modelcanvas/

\section{Debemos responder, por lo ranto, con un enfoque didáclico integral, por lo que el resultado del proceso de aprendizaje debe ser un equilibrio entre "la cabeza, el corazón y la mano"}

deben modificar las formas de gestión de los proyectos.

Esta nueva visión de los proyectos, cuya característica es su agilidad, tiene como objetivo dar garantías a las demandas principales de la industria en la que se ha generado: valor, reducción del tiempo de desarrollo, agilidad y fiabilidad.

La gestión ágil es necesaria en mercados rápidos. Por lo tanto también será necesaria en la sociedad actual, en la búsqueda de empleo, teniendo en cuenta la flexibilidad y versatilidad del mercado laboral.

La gestión ágil de mi proyecto profesional no es una gestión de anticipación (requisitos, diseño, planificación y segui- 


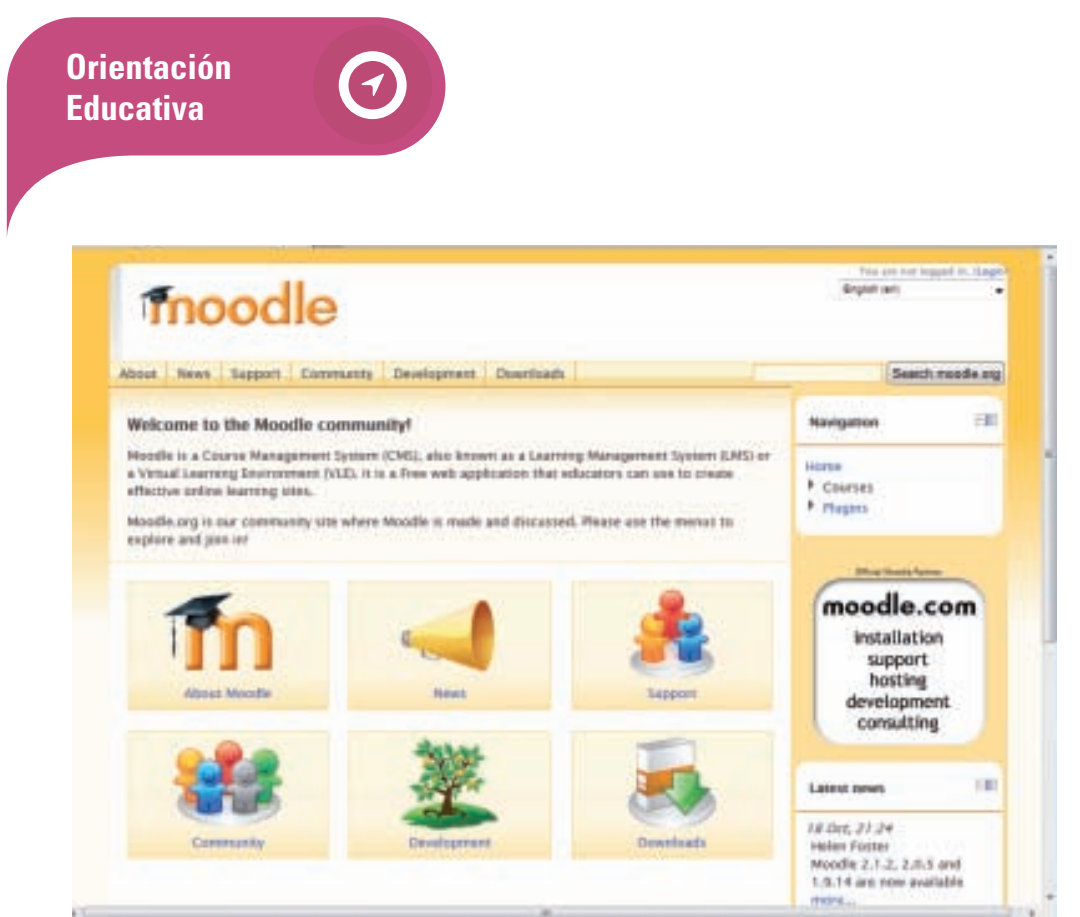

Aula Moddle.

Fuente: https://moodle. org/?lang=es miento) sino de adaptación (visión, exploración y adaptación).

Las actividades o acciones más relevantes que se han desarrollado a lo largo del programa han sido:

入 Análisis del estado actual de la situación.

入 Formación del profesorado (metodologías ágiles, learning by doing).

$\boldsymbol{\lambda}$ Implantación del proyecto en centros piloto.

入 Análisis, mejora y desarrollo a todos los centros de Formación Profesional de la Comunidad Foral de Navarra.

\section{Evaluación y/o discusión de una experiencia piloto en la Comunidad Foral de Navarra}

La evaluación de este programa anima a su aplicación y desarrollo para realizar un programa modelo que pueda ser aplicable en los distintos ámbitos en los que se requiera el fomento del emprendimiento y la empleabilidad.

En esta primera fase en la que nos encontramos se ha realizado un análisis sobre la percepción del alumnado de las diferentes competencias, como base para desarrollar mejoras al programa y establecer en próximos cursos mediciones de las competencias en el entorno empresarial, una vez hayan accedido a la empresa.

Se realiza una comparativa entre el grupo de control y el grupo piloto (experimental), tras la cual se evaluará la técnica a utilizar que puede resultar más adecuada. Las escalas Likert tienen la doble vertiente de poder ser consideradas categóricas o numéricas, por lo que dependiendo de los resultados de la parte descriptiva se utilizará la que se considera más apropiada. Así, si se consideran como numéricas se utilizará "T" de Student y si se considera su tratamiento como categóricas sería más adecuado utilizar el test Chi-cuadrado.

También se realiza un estudio entre las variables que describen la muestra y las competencias, la técnica a utilizar estará en función del comportamiento de los resultados descriptivos.

Una vez desarrollado el análisis estadístico de la percepción del alumnado se han detectado diferencias significativas en tres variables importantes.

त Iniciativa. La media de los participantes que sí han cursado EIE con este programa es significativamente más alta (4.16) que la media de los que de cuasi-control (3.76) con $\mathrm{P}<.05$ en el test de Student y aún más con $P<.01$ en la alternativa no paramétrica. El tamaño del efecto es pequeño $(<.10)$.

त Confianza en sí mismo. De nuevo la media de los casos cuasi-experimentales es significativamente mayor (3.60) que la media de los cuasi-controles (3.12) con $\mathrm{P}<.05$ en ambos test estadísticos aunque con tamaño del efecto pequeño.

入 Comunicación. Una vez más la media de los sujetos participantes en el curso EIE es significativamente más elevada (3.85) que las media de los que no han asistido (3.41) con $\mathrm{P}<.05$ en el test de Student y con $\mathrm{P}<.01$ en la alternativa no paramétrica aunque con tamaño del efecto pequeño.

\section{Conclusiones}

La permanente evolución y transformación del mercado laboral y de la formación profesional requiere de nuevas formas de relación entre el sistema educativo y el sistema productivo basándose en los pilares fundamentales que se recogen en la normativa europea y española: la gestión de calidad, la empleabilidad y las nuevas tecnologías.

Tras la aplicación, medición y análisis del programa llevado a cabo en varios 


\section{La permanente evolución y Iransformación del mercado laboral y de la formación profesional requiere de nuevas formas de relación entre el sistema educativo y el sistema productivo}

centros de la Comunidad Foral de Navarra, podemos determinar que el programa desarrollado basado en metodologías ágiles y la plataforma MOODLE, al menos mejora significativamente 3 capacidades consideradas esenciales en la empleabilidad de los jóvenes: la iniciativa, la confianza en sí mismo y la comunicación.

También se ha fomentado el uso de las $\mathrm{TIC}$, que se considera una competencia imprescindible en la sociedad actual.

Si seguimos el proceso de Gestión de Calidad desarrollado en el ciclo de Deming (figura y con la finalidad de asegurar y mejorar el programa utilizado deberemos actuar en las áreas específicas en las que queremos mejorar, dotando al programa de actividades que mejoren las competencias críticas de empleabilidad en las que no hemos encontrado mejoras significativas con el programa, teniendo en cuenta que puede considerarse un éxito la mejora significativa del alumnado de formación profesional en tres de las 10 competencias con la aplicación de un programa curricular •

\section{HEMOS HABLADO DE}

\section{Búsqueda de empleo, metodologías ágiles, orientación profesional, emprendimiento, formación profesional.}

Este artículo fue solicitado por PADRES y MAESTROS en diciembre de 2014, revisado y aceptado en mayo de 2015.

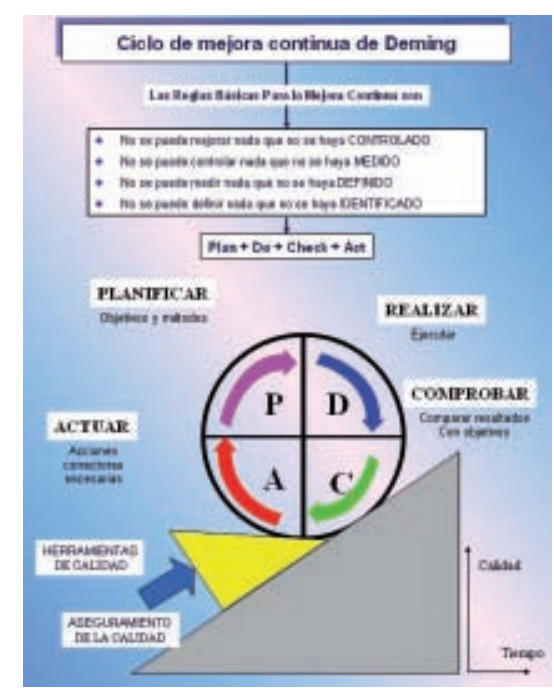

Mejora continua de Deming

Fuente: http://endrino. pntic.mec.es/jhem0027/

calidad/sistema/ sistema.html

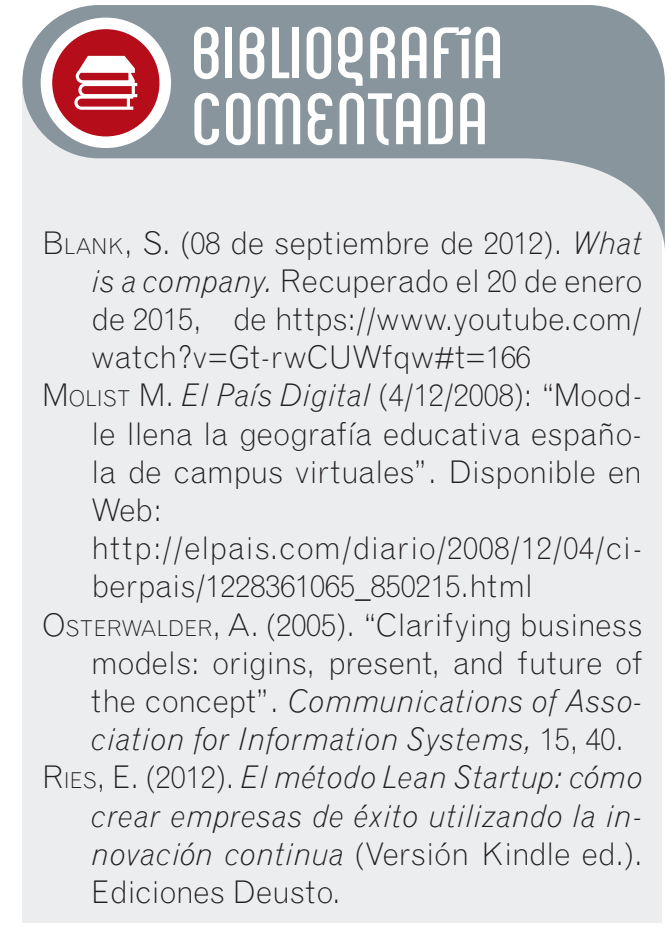

Aprende como emprender: https://www.youtube.com/watch?v=MsmPPQ6kAEU

Busniness model canvas: http://www.businessmodelgeneration.com/canvas/bmc

Actividad de aplicación: https://www.youtube.com/watch?v=i1Le5GYkBT8

Presentación programa formativo: https://www.youtube.com/watch?v=NXPKt2zylQA. Actividad creación de prototipos: https://www.youtube.com/watch?v=XoV_DT1vE74 https://www.youtube.com/watch?v=XoV_DT1vE74. 